\title{
LOS MODELOS DE GÉNERO DESDE UNA PERSPECTIVA INTERCULTURAL: UN PROYECTO DE INVESTIGACIÓN PARA EL DESARROLLO DE POLÍTICAS DE GÉNERO EN EL ÁMBITO UNIVERSITARIO EN ITALIA, ESPAÑA Y ARGENTINA.
}

\author{
Gender models from an intercultural perspective: a research project on the \\ development of gender politics in italian, spanish and argentinian universities.
}

\author{
Chiara Santoro \\ $M^{a}$ Carmen Monreal Gimeno
}

Fecha recepción: 13/07/2016

Fecha aceptación: 02/11/2016

RESUMEN: En este articulo se pretende dar cuenta de los fundamentos teóricos, de las herramientas metodológicas y de las hipótesis planteadas en la investigación "Los modelos de género entre el alumnado universitario desde una perspectiva intercultural". Esta investigación, que es parte del proyecto europeo IRSES Gendercit, tiene como objetivo el estudio desde una perspectiva intercultural de los actuales modelos de género en las y los estudiantes universitarios/as de Italia, España y Argentina. En primer lugar, se presenta el posicionamiento teórico sobre el cual se basa esta investigación: el género comprendido desde una postura relacional y transversal. Además, se destaca la importancia desde la intervención en las políticas de género en ámbito universitario, del desarrollo de herramientas de medida y de análisis de la resistencia de modelos de género fijos y normativos. Por ello, se presenta el cuestionario sobre modelos de género y los resultados esperados a partir de su aplicación. Para concluir, se pondrá en evidencia como entender el género como un elemento relacional supone salir de un análisis que se concentre solo sobre datos numéricos y porcentajes, abarcando una realidad mas compleja, que nos permite generar herramientas reales para el cambio, especialmente en ámbito universitario.

PALABRAS CLAVE: igualdad de género, estudiantes universitarios, evaluación continua, análisis comparativo

ABSTRACT: This article aims to present the theoretical foundations, methodological tools and research hypotheses of the research "gender models at the university level from an intercultural perspective." This research, which is part of the European project IRSES Gendercit, aims to study from an intercultural perspective current gender models in university students in Italy, Spain and Argentina. First, we will present the theoretical position on which this research is based: the concept of gender understood from a relational and transversal approach. Moreover, we will stand out how the development of measurement tools to analyse the resistance of gender fixed models is important to increase university gender policies. Then, we present the questionnaire on gender models and the expected results from its application. To conclude, we will focus on how to understand gender as a relational concept, it means to go out from an analysis that concentrates only on numerical data and percentages. This position allows us to cover a more complex reality, and generate real tools for change, especially at higher education level. 

analysis.

KEYWORDS: gender equality, university students, continous evaluation, comparative

\section{Introducción}

En este artículo se pretende presentar los supuestos teóricos, metodológicos, y los resultados esperados/hipótesis del proyecto de investigación "Modelos de género entre el alumnado universitario desde una perspectiva intercultural". Esta investigación nace de diferentes presupuestos: una postura teórica bien definida, que apuesta por una comprensión del concepto de género como relacional, y las reflexiones criticas desarrolladas en el trabajo de intervención para el desarrollo de políticas de género a nivel universitario en España, en particular en la Oficina para la igualdad de la Universidad Pablo de Olavide de Sevilla. Además, gracias a la inclusión de la investigación en el proyecto europeo IRSES Gendercit (género y ciudadanía), ha sido posible integrar la perspectiva intercultural, que nos permite contrastar la resistencia de los modelos en contextos universitarios de diferentes países, Italia, España y Argentina, además de analizar las buenas practicas realizadas para conseguir la igualdad substancial.

A partir de estos presupuestos, y dada la escasez de estudios comparados alrededor de la cultura y de los modelos de género especialmente en el espacio superior de educación, se creyó necesario realizar un estudio sobre la resistencia de modelos de género tradicionales, que partiera de una visión amplia del concepto de género y se enfocara principalmente en las relaciones entre los géneros, en las que están implicados/as los/las estudiantes universitarias, para comprender e incluir en las políticas publicas de género en ámbito educativo elementos que pudieran apoyar la consecución de la igualdad substancial entre mujeres y hombres, que evidentemente no puede pasar exclusivamente a través de la inclusión de las mujeres en el ámbito universitario.

Estos elementos se presentan en el primer apartado, estado de la cuestión, que a su vez se divide e dos sub-apartados. En el primero, se presentan algunas observaciones críticas llevadas a cabo a partir de la observación directa y del trabajo de intervención en la Oficina para la Igualdad de la Universidad Pablo de Olavide de Sevilla y del desarrollo y evaluación continua de las políticas universitarias en ámbito universitario.

Desde un análisis directo del desarrollo, de la implementación y de la evaluación de las políticas de género en ámbito universitario, se detecta la necesidad de integrar el diagnostico de la situación de igualdad entre hombres y mujeres con datos que nos permitan comprender la resistencia entre los/las estudiantes de los modelos de género normativos de masculinidad y feminidad que, como hemos visto, constituyen las mismas bases que sustentan la reproducción de un sistema de poder patriarcal y afectan directamente a la igualdad sustancial entre mujeres y hombres.

En el segundo, se presenta el concepto de género como concepto relacional, poniendo en evidencia como sólo alejándonos de una postura que utilice el termino género como sinónimo de mujer podemos comprender y cambiar las estructuras de poder patriarcales. El género se comprende como una característica de la identidad individual, pero al mismo tiempo, esta identidad se define de forma complementaria y excluyente respecto al otro/ a la otra, y a sus representaciones socio-culturales, influyendo en las elecciones, expectativas, roles individuales, a través de modelos fijos de masculinidad y feminidad.

En el tercer apartado, método, se presentan los objetivos del estudio y la nueva herramienta metodológica, el cuestionario sobre modelos de género, que ha sido utilizada en 
esta investigación. Se presenta la muestra elegida y las modalidades de distribución y análisis de los datos, que integra la perspectiva intercultural.

En los resultados esperados del estudio, se analizan los resultados de la prueba piloto del cuestionario realizada en la Universidad Pablo de Olavide y las hipótesis de la investigación que de allí se desprenden, además de los supuestos que justifican la perspectiva intercultural del mismo.

Por ultimo, en la discusión y conclusiones, se presentan las críticas y los puntos fuertes de la herramienta metodológica ya implementada, además de dar cuenta de sus posibles aplicaciones en el ámbito de la intervención para el desarrollo de las políticas universitaria orientadas a conseguir la igualdad substancial entre hombres y mujeres.

\section{Estado de la cuestión: un enfoque desde la intervención y un posicionamiento teórico para la comprensión y el análisis de los modelos normativos de género, desde una perspectiva intercultural}

En este primer apartado se presentan los dos puntos de partida que han fundamentado la necesidad del desarrollo de la investigación "Modelos de género entre el alumnado universitario desde una perspectiva intercultural". En primer lugar, se realiza un análisis de las políticas de género en el ámbito universitario a partir de la observación y del trabajo realizado en la oficina para la igualdad de la Universidad Pablo de Olavide; en segundo lugar, se determina un posicionamiento teórico preciso que concibe el género como un concepto relacional y, los modelos de género, como elementos de diagnóstico fundamentales en el desarrollo de la investigación y como elementos de intervención desde una perspectiva de género.

Además, la posibilidad de integrar la investigación en el proyecto europeo IRSES GENDERCIT ha permitido ahondar, tanto en el significado de intervención para la igualdad (especialmente en el ámbito universitario), como en el mismo concepto de género y en los matices en los que se produce este concepto en los diferentes contextos culturales. El proyecto IRSES GENDERCIT tiene el objetivo de favorecer e implementar el intercambio de conocimiento y la investigación en la temática de género e igualdad entre investigadoras/es latinoamericanas/os y europeas/os; por esta razón y, a partir del mismo recorrido personal/social/cultural de las investigadoras implicadas en esta investigación que ofrecía un conocimiento situado y una mirada desde y entre las fronteras, se elige abarcar una muestra intercultural que comprenda tres países: Italia, España y Argentina. Por un lado, esta posibilidad permite integrar a la investigación una mirada a la complejidad que conlleva integrar conceptos considerados universales a partir del discurso de las organizaciones internacionales y de las declaraciones universales (como igualdad, género o educación para los derechos humanos) con contextos específicos, reconociendo el peso de las culturas, de las historias socioculturales de los pueblos y de sus movimientos políticos. Por otro lado, la perspectiva intercultural que se abarca en esta investigación pretende ir más allá de una mera comparación entre países para enfocarse en el intercambio de buenas prácticas (tanto en el ámbito de la intervención, así como en el de la investigación) y enfocarse, también, en la necesidad de la comprensión de los procesos locales para la implementación de políticas de género eficaces. Desanclándose de un enfoque culturalista, la perspectiva intercultural permite también evidenciar el tema de la transversalidad e internacionalización de una estructura de poder patriarcal que sigue reproduciendo culturas de género que se fundamentan en modelos de género binarios, excluyentes y complementarios, que transcienden las fronteras nacionales y se entrelazan con las mismas estructuras de poderes, de las cuales las universidades representan un papel de tornasol. 


\subsection{Políticas de género y diagnostico de la situación de igualdad: los modelos de género como medida de igualdad sustancial.}

La investigación "Modelos de género entre el alumnado universitario desde una perspectiva intercultural" se ha fundamentado en el estudio de las políticas de género en el ámbito universitario español y sobre la observación directa y critica del desarrollo, implementación y evaluación de las políticas de género en la Oficina para la Igualdad de la Universidad Pablo de Olavide de Sevilla.

Desde el año 2007, las Universidades españolas, en cuanto forman parte de la administración pública, tienen que cumplir con las políticas integrales de género que se establecen a nivel estatal con la Ley 3/2007, de 22 de Marzo, para la igualdad efectiva de mujeres y hombres. La disposición adicional duodécima de la LOM-LOU (Ley Orgánica 4/2007, del 12 de abril, por la que se modifica la Ley Orgánica 6/2001, de 21 de diciembre, de Universidades) obliga a la constitución de unidades de género en las universidades públicas. Desde esta perspectiva, ya en el año 2008 se constituye la Oficina para la igualdad, una unidad funcional de la estructura administrativa de la universidad, y órgano permanente para el desarrollo de las políticas de igualdad de la Universidad Pablo de Olavide, además de ser responsable de la aplicación de las leyes estatales de género en la misma Universidad.

Las políticas de género en el ámbito universitario se recogen en un Plan de Igualdad, que cada Universidad española está obligada a desarrollar a partir de un diagnostico previo de la situación de igualdad entre hombres y mujeres, como se establece en el artículo 45 de la Ley 3/2007. Estas políticas derivan directamente de los elementos que considera el diagnóstico, y de los datos que allí se incluyen.

El diagnóstico realizado en la Universidad Pablo de Olavide recoge datos cuantitativos sobre la posición de mujeres y hombres en los diferentes ámbitos de la Universidad, además de un estudio cuantitativo sobre la experiencia de la conciliación vida familiar-vida laboral. A partir de un análisis crítico del diagnostico y de las políticas incluidas en el Plan de igualdad, se pueden destacar diferentes observaciones, que están a la base de la elaboración de los objetivos de esta investigación:

- Los datos cuantitativos (porcentajes) sobre la distribución de hombres y mujeres (alumnado, profesorado, personal administrativo y de servicio) recogidos en el diagnostico no son suficientes para comprender la situación real de igualdad, ya que esta no se fundamenta exclusivamente sobre una distribución homogénea de los dos sexos en los diferentes cargos, en las diferentes áreas, y las diferentes ramas de estudio, sino sobre el cambio, tanto en hombres como en mujeres, de creencias y modelos que fundamentan prácticas sexistas y discriminatorias.

- A causa de la falta de datos recogidos en el diagnóstico que nos permitan comprender las necesidades reales, no se evidencia en el Plan de Igualdad cuáles deberían ser los contenidos de las acciones de formación en género que se pretenden desarrollar, y sus procedimientos de evaluación.

A partir de estas observaciones, y de la convicción de que la Universidad es un ámbito de intervención fundamental para la consecución de la igualdad sustancial entre hombres y mujeres, nace la necesidad de desarrollar un diagnostico de la situación de igualdad que permita comprender el estado actual de los modelos de género de masculinidad y de feminidad.

Integrar los modelos de género en el diagnostico de la situación de igualdad en el ámbito universitario, nos permite comprender el género desde una dimensión más compleja y relacional, y profundizar en el significado mismo de políticas de género, de los sujetos 
destinatarios, y de los objetivos que nos proponemos cuando hablamos de igualdad sustancial entre hombres y mujeres.

\subsection{El género como concepto relacional: un abordaje en la investigación desde una perspectiva de género}

El término género se utiliza por primera vez en el ámbito médico, en 1955, gracias a los estudios del doctor John Money. En esta primera definición, Money explica el rol de género como "todo cuanto una persona dice o hace para indicar a los demás o a sí misma el grado en que es niño o varón, niña o mujer respectivamente." (citado en Garcia y Freire, 2003)

Aunque esta primera definición carece de la precisión conceptual que este concepto de género empezó a tener con las teorizaciones feministas, ya expresa perfectamente una de sus principales variables: su carácter relacional. De hecho, pensar en el género permite ampliar el enfoque y pasar a un nivel relacional de análisis del fundamento y de las estructuras del mismo sistema patriarcal; significa comprender cómo las consecuencias de este sistema de privilegios recaen, aunque de forma desigual, sobre la sociedad en su conjunto, creando un sistema de valores, normas y conductas sobre la base de una dicotómica división entre los cuerpos sexuados. Además, el concepto de género nos permite también comprender la persistencia y capacidad de reproducirse del sistema patriarcal, ya que esta está estrechamente relacionada con su forma de influir internamente, caracterizar y limitar cada aspecto de la vida humana y de las relaciones entre personas, gracias a la creación de modelos fijos de género, de masculinidad y feminidad ${ }^{1}$.

Por un lado, como afirma Garaizabal, el género es una de las variables fundamentales de la definición de la identidad y "utiliza un proceso de reflexión y observación simultaneas que tiene lugar en todos los niveles del funcionamiento mental y por medio del cual el ser humano se juzga a sí mismo en función de cómo cree que le juzgan los otros comparándoles con ellos y con respecto a una tipología significativa para estos" (Valcuende y Blanco, 2003). Por el otro lado, la identidad se define como un flujo constante de informaciones y de intercambios simbólicos entre el individuo y los demás, en un proceso sin fin de observación, comprensión, juicio, adaptación o rechazo. Un proceso que se define por una oposición fundamental, en un sistema social y cultural completamente determinado por la división entre los sexos y por el patriarcado, que enfrenta lo masculino y lo femenino. Como describe el modelo congruente, la masculinidad y la feminidad se perciben como una realidad bipolar opuesta, como extremos opuestos, excluyentes y complementarios. (García y Freire, 2003).

Como explica Bourdieu (2000), "al carecer de otra existencia que la relacional, cada uno de los dos sexos es el producto del trabajo de construcción diacrítica, a un tiempo teórico y práctico, que es necesario para producirlo como cuerpo socialmente diferenciado del sexo opuesto (desde todos los puntos de vista culturalmente pertinentes), es decir, como habito viril, por consiguiente, no femenino, o femenino, por consiguiente, no masculino."

Esta comprensión relacional del concepto de género es clave para salir de una lógica dual y de oposición, que enfrenta a hombres y mujeres y los individualiza como extremos incompatibles, una conceptualización que a veces había sido utilizada también en los primeros análisis teóricos de los estudios de mujeres. Como afirma Maccoby, la cuestión es considerar si la masculinidad y la feminidad son dos polos opuestos o, por el contrario, debemos entender que ambas dimensiones se influyen y condicionan mutuamente (citado en

\footnotetext{
1 por modelos de género entendemos el conjunto de valores, conductas, aptitudes y expectativas que se consideran propias para un hombre y para una mujer en un contexto y una época determinada, y que están a la base de relaciones desiguales y de poder, que fundamentan y siguen reproduciendo el sistema patriarcal.
} 
Monreal y Martínez, 2010). De esta forma, el concepto de género empieza a alejarse desde su base dual, diferenciándose verdaderamente del concepto de sexo, para abrirse como una categoría más comprensiva, que abarcara también construcciones socio-identitarias y formas de socialización que surgen de la categorización hombres-mujeres, pero a la vez la superan, gracias, al debate a cerca de la androginia o de las personas transgénero (Casares, 2006).

Considerar al género en toda su amplitud de significación es uno de los elementos claves para comprender la igualdad y esperar un verdadero cambio de la estructura social que pueda permitir alcanzar la igualdad.

\section{Método: el cuestionario sobre modelos de género en el ámbito universitario}

A partir de la confluencia entre el posicionamiento teórico presentado y las observaciones criticas sobre el desarrollo de políticas de género en la Oficina para la Igualdad de la Universidad Pablo de Olavide de Sevilla, deriva la necesidad de desarrollar una investigación que explore la resistencia de modelos de género de masculinidad y feminidad hegemónicos entre estudiantes universitarios/as, como uno de los principales elementos de la persistencia de una estructura patriarcal. Además, la inclusión de la investigación en el proyecto europeo IRSES Gendercit, ha permitido ampliar el horizonte nacional de este planteamiento, incluyendo en este análisis la perspectiva intercultural.

\subsection{Preguntas y objetivos de la investigación}

A partir de los elementos considerados, surge el objetivo general de la investigación que es: "Analizar los modelos de género existentes desde una perspectiva intercultural en la población universitaria de Argentina, Italia y España".

Este objetivo es el resultado de dos preguntas de investigación que se han ido planteado a partir de la revisión bibliográfica y de la observación en el trabajo de intervención presentada:

- ¿Existe una resistencia de modelos de género de masculinidad y feminidad entre los/las estudiantes universitarios/as?

- ¿Existen diferencias en la resistencia de modelos de género normativos de masculinidad y feminidad entre los/las estudiantes de Argentina, Italia y España?

Para alcanzar el objetivo general y responder a las preguntas de investigación formuladas se han desarrollado diferentes objetivos específicos, que se reflejan en la misma estructura del cuestionario presentado a continuación, que van ahondando en los diferentes ámbitos donde se reproducen de forma transversal los modelos de género. Los objetivos específicos son los siguientes:

- Autopercepción y percepción de los demás: analizar la autopercepción de estudiantes universitarios en los tres países considerados en el estudio (Argentina, Italia, España) y como perciben que los demás les perciben.

- Ámbito educativo: Estudiar las relaciones de género en el ámbito educativo en los tres países considerados en este estudio (Argentina, España, Italia).

- Uso del tiempo. Estudiar las relaciones de género en el uso del tiempo y en las actividades desarrolladas en función del sexo en los tres países considerados en el estudio (Argentina, Italia, España). 
- Conciliación ámbito laboral/familiar/personal: Analizar la conciliación entre el ámbito personal/ familiar/laboral en función de sexo en los tres países considerados en el estudio (Argentina, Italia, España).

- Relaciones afectivos/sentimentales/sexuales: Analizar las relaciones afectivosentimentales-sexuales en función del sexo en los tres países considerados en el estudio (Argentina, Italia, España).

Podemos afirmar que, a partir de esta estructura, toda la investigación se fundamenta sobre dos ejes teóricos/conceptuales/metodológicos:

- El análisis de modelos de género como variable fundamental para la comprensión de la situación de igualdad entre hombres y mujeres en el ámbito universitario.

- La integración de la perspectiva intercultural como elemento clave para la formulación de posibles hipótesis sobre las causas que pueden conllevar una mayor o menor flexibilidad de los modelos de género integrados por el alumnado.

\subsection{Metodología y herramienta de medida: el cuestionario sobre modelos de género}

A partir de estos ejes se ha elegido una metodología cuantitativa, para indagar en la resistencia de modelos de género fijos. La elección de esta metodología ha estado directamente relacionada con el enfoque en la intervención y en el desarrollo de nuevas herramientas para la implementación de políticas de género de esta investigación.

De hecho, la construcción de una herramienta cuantitativa que permita recoger datos sobre resistencia de modelos de género permitiría la inclusión de esta variable en el análisis previo (diagnostico) al diseño de políticas de género en el ámbito universitario.

Por el otro lado, es evidente que medir una variable tan compleja y transversal como los modelos de género implica ser conscientes de las aproximaciones llevadas a cabo por parte de los/las investigadores/as en la creación de la herramienta. A partir de esta realidad el cuestionario se propone reflejar una concepción del género y de los modelos compleja, intentando dar cuenta de la multiplicidad de facetas y contextos donde estos se explican, además de dejar espacios de reflexión personal y la posibilidad de expresarse entre varias opciones a los/las entrevistados/as.

Para dar voz a esta multiplicidad y complejidad, sin perder el valor de los modelos de género como posible variable de medición de la situación de igualdad substancial entre hombres y mujeres se han utilizado tres diferentes estrategias, que corresponden a características propias de los modelos de género.

- Los modelos de género son transversales, y se manifiestan en los diferentes ámbitos de la vida de cada uno/ cada una de nosotros, y en las relaciones que establecemos en los diferentes contextos con los demás.

Por esto, el cuestionario se compone de 6 apartados que corresponden a las diferentes esferas donde se manifiesta la adhesión a modelos hegemónicos de referencia, y a los objetivos específicos planteados: autodefinición y percepción de los demás, ámbito educativo, uso del tiempo, conciliación ámbito laboral/familiar/personal, relaciones afectivos/sentimentales/sexuales.

- "Los modelos de género son mapas por los cuales transitamos" (Valcuende y Blanco, 2003). La existencia de modelos de referencia en los diferentes contextos y culturas no significa que nuestras experiencias sean una mera repetición de estos contenidos que recibimos del exterior. 
Como hemos visto, el género es también una variable individual, que se articula como una característica identitaria propia, en constantemente relación con otros elementos y con las elecciones individuales de cada uno/cada una. Para dar la posibilidad de expresar estos matices, cada apartado del cuestionario se divide a su vez en dos subapartados, el primero con ítems a los que se tiene que contestar según las experiencias personales, mientras en el segundo se pide expresar opiniones y creencias. Esta estrategia, además de restituir la complejidad de la adhesión a los modelos de género, permite evitar o por lo menos comprender respuestas que sigan el discurso de acuerdo con lo "políticamente correcto" sobre la igualdad de género.

- Los modelos de género afectan tanto a hombres como a mujeres y se establecen sobre una base relacional. Por este motivo, el cuestionario indaga la resistencia de ambos modelos de masculinidad y de feminidad en chicos y chicas, ya que tanto la persistencia de una visión estereotipada hacia si mismo/a como hacia el otro/la otra conllevan la reproducción de roles, expectativas, oportunidades diferenciadas por sexo.

En todos sus apartados el cuestionario utiliza escalas Likert, a través de las cuales el encuestado/la encuestada puede expresar su grado de acuerdo y desacuerdo en una escala de 1 a 4. Además, para incluir espacios de expresión de las apreciaciones individuales de los diferentes modelos, al final de cada apartado se encuentra un espacio libre donde el encuestado/la encuestada pueden explicar si se han encontrado cómodo/a en la recopilación.

\subsection{Muestra y modalidades de distribución del cuestionario}

La muestra elegida ha sido de 600 estudiantes universitarios pertenecientes a tres diferentes universidades, en particular 200 estudiantes argentinos/as (Universidad de Cuyo, Mendoza), 200 italianos/as (Universitá degli studi di Firenze) y 200 españoles/as (Universidad Pablo de Olavide, Sevilla).

La muestra es descriptiva, post facto, correlacional e intercultural. Se han incluido en la muestra estudiantes de diferentes carreras, dividiendo la muestra en ramas: por cada universidad, se han incluido 100 estudiantes de la rama de ciencias duras y 100 estudiantes de la rama de ciencias sociales/humanidades. La inclusión de este elemento permite dar cuenta de las diferencias existentes en el mismo ámbito universitario, que reproduce a menudo en la estructura y en sus características la difusión sectorial en la sociedad en su conjunto de ciertas temáticas y practicas, como las relativas a la igualdad de género. La atención puesta en este elemento ha permitido también llegar a una muestra equilibrada por sexo, ya que sigue existiendo una división muy fuerte y transversal entre la presencia de hombres y mujeres en las diferentes carreras y facultades.

Aunque la compilación de los cuestionarios sea autónoma por parte de los/las encuestados/as, la presencia y la observación directa por parte de una investigadora en el momento de la distribución facilita la resolución de dudas y la correcta compilación del cuestionario. Además, esto permite la recogida de elementos sobre los contextos, fundamentales en una investigación intercultural, y la recolección de opiniones, ideas, sugestiones, debates alrededor de la temática, desencadenados por el mismo cuestionario, material que permite desarrollar diferentes tipos de reflexiones útiles a la hora de interpretar los datos. 


\section{Resultados esperados: la prueba piloto en la Universidad Pablo de Olavide, Sevilla, España.}

El mes de junio de 2013 se llevó a cabo en la Universidad Pablo de Olavide de Sevilla una prueba piloto para evaluar el cuestionario, que involucró 40 estudiantes de postgrado, hombres y mujeres. Los resultados de la misma, aunque claramente no significativos a nivel estadísticos, justifican la necesidad de esta investigación y nos dan pistas relativas a los resultados esperados y las hipótesis planteadas en la misma.

En particular, a partir de la prueba piloto se destaca que:

- En las experiencias personales de los/las encuestados/as se detecta un cierto rechazo de los modelos normativos

- En las opiniones generales se evidencia una resistencia de los modelos de género, sobre todo en los hombres, tanto del modelo de masculinidad como en el de feminidad normativa.

- Se evidencia la reproducción de modelos de géneros excluyentes y a la vez complementarios, en cuanto se destaca del análisis de los datos la persistencia de espacios diferenciales de socialización entre hombres y mujeres, sobre todo en la gestión de las actividades relativas al tiempo libre y en el ámbito educativo, y, por otro lado, una fuerte carga emotiva y de dependencia en las relaciones de pareja.

En resumen, los resultados de esta primera prueba piloto parecen evidenciar una cierta resistencia al cambio de los modelos en las opiniones, especialmente fuerte en los hombres, que coexiste con un desdibujamiento de referencias normativas en las experiencias personales de los/las estudiantes.

Comparando estos resultados con la observación directa realizada en las distribuciones presenciales del cuestionario en las aulas universitarias argentinas, españolas e italianas y con los comentarios de los diferentes momentos de discusión con los grupos de investigación locales y con estudiantes de las universidades involucradas en el proyecto, podemos afirmar que los resultados esperados por esta investigación se reflejan en las hipótesis planteadas, que son las siguientes:

- Existen fuertes resistencias de los modelos normativos de masculinidad y feminidad en los tres países.

- Existe una mayor resistencia de los modelos de género normativos en los hombres que en las mujeres.

- Es mayor la resistencia de los modelos de género normativos en los discursos y en las ideas generales que en las practicas y experiencias individuales de los/las estudiantes

- Existen diferencias entre los/las estudiantes de los tres países respecto a los ámbitos de mayor resistencia de los modelos de género (ámbito personal, educativo, familiar, laboral, en el uso del tiempo libre, en las relaciones de pareja, en las relaciones sexuales)

Además, a partir de la revisión bibliográfica y de la observación directa realizada en cada país involucrado en el estudio, se prevé que la influencia de la historia de los movimientos de mujeres y de los feminismos, y del proceso de institucionalización de publicas para la igualdad, genere diferencias significativas respecto a la resistencia y los contenidos de los mismos modelos prevalentes. Este hecho, si es demostrado, posibilitaría evidenciar la importancia tanto de las luchas políticas feministas como de las políticas publicas en este ámbito para la consecución de la igualdad substancial entre mujeres y 
hombres, a través del cambio de la cultura de género prevalente. Además, a través de la comparación entre países, se destacaría la necesidad de integrar estrategias y acciones locales en una dimensión más internacional, sin perder la necesaria comprensión de los contextos e incluir una evaluación continua de los procesos.

\section{Discusión y conclusiones}

Como hemos visto, la finalidad de la investigación es el análisis de los actuales cambios y persistencia de los modelos de género patriarcales, para fundamentar el desarrollo de políticas de género universitarias a partir de una perspectiva relacional y teniendo en cuenta las resistencias, los roles y los estereotipos de género. El trabajo de distribución de la herramienta de medida y la posibilidad de presenciar todas las fases del proceso, conociendo de cerca los diferentes contextos universitarios involucrados en los diferentes países, gracias a la inclusión de la investigación en el proyecto europeo IRSES Gendercit, ha permitido poner en evidencia los puntos fuertes y débiles de la misma.

Si por un lado, la recolección de estos datos sobre modelos de género ha suscitado interés en las diferentes universidades y en el alumnado, estimulando el debate y la comprensión de las temáticas tratadas en los diferentes países, por otro lado, los diferentes posicionamiento teóricos, políticos, normativos y sociales alrededor de la lucha para la igualdad de género han evidenciado la dificultad de conseguir una comprensión realmente integral de la resistencia de modelos fijos de género exclusivamente a través de la distribución de la herramienta, sin tener en cuenta variables socio-culturales especificas y sin incluir elementos cualitativos en la investigación. Desde este punto de vista, nos proponemos integrar en futuras investigaciones estas metodologías, incluyendo grupos de discusión y entrevistas en profundidad, además de un estudio intercultural sobre normativas y políticas de género desarrolladas en los distintos países.

En todo caso, medir los modelos de género es un primer paso para comprender el enraizamiento real del sistema y de las creencias patriarcales. Además, centrarse en el ámbito universitario supone poner bajo observación una de las estructuras que rigen nuestra sociedad, donde se construye el conocimiento científico, y en la cual transitan hombres y mujeres que ya han sido socializados por distintos factores (escuela, familia, grupo de pares, comunidad, medios de comunicación) pero al mismo tiempo han tenido la posibilidad de ser influenciados por múltiples recorridos personales y haber desarrollados recursos propios.

Por todo ello, analizar los modelos de género normativos en la población universitaria significa comprender en qué medida el sistema sexo-género continúa afectando en las elecciones y expectativas individuales, más allá de los conocimientos adquiridos, los recursos individuales y la difusión y visibilización de modelos no conformes al estereotipo.

Apostar por la libertad individual, por la libre expresión de si mismo/a, significa, antes que todo, comprender y ser conscientes, tanto hombres como mujeres, de la carga de la socialización que sigue determinando una lectura de nuestras experiencias pasadas y futuras en continuidad con nuestros atributos sexuales. Al mismo tiempo, comprendiendo el concepto de género a partir de una postura fluida y relacional, las personas tienen un potencial transformador de estas mismas coordenadas de referencia que codifican su ser social. Por esto, es fundamental tener en cuenta en esta investigación, y en general la investigación de género, su función de comprensión de los limites vigentes en el sistema social, pero, al mismo tiempo, entenderla como una oportunidad para dar voz a una realidad que muchas veces supera estos confines fijos, creando nuevos mapas o perdiendo la necesidad de mapas en los cuales transitar: una realidad hecha por hombres y mujeres en devenir, que luchan entre sus 
experiencias de discriminación, de adhesión y de ruptura, buscando formas de afirmarse mas allá de los modelos fijos de referencia, y en espacios que el sistema educativo tiene la obligación de asegurar.

\section{Referencias bibliográficas}

Barberá, E., Martínez, I. (coords.) (2005). Psicología y género. Madrid: Pearson education.

Beauvoir, S. (1981) El segundo sexo. Madrid: Aguilar Ediciones.

Bellucci, M. (1994) De los estudios de la mujer a los estudios de género: han recorrido un largo camino. En Fernández, A. (Eds.) La mujer en la imaginación colectiva, Barcelona: Paidos

Beltrán, E., Maquieira,V. (coords) (2012). Feminismos: debates teóricos contemporáneos. Madrid: Alianza. III ed.

Biemmi, I. (2012), Educare alla parità, Roma: Conoscenza.

Bourdieu, P. (2000) La dominación masculina. Barcelona: Editorial Anagrama.

Cagnolati A., Pinto Minerva F., S. Ulivieri (Eds.) (2013). Le frontiere del corpo. Mutamenti e metamorfosi.Pisa: ETS

Campani G. (2000). Genere, etnia e classe. Migrazioni al femminile tra esclusione e identità, Pisa: ETS.

Campani G. (2009). Veline, nyokke e cilici. Femministe pentite senza sex e senza city. Bologna: Odoya.

Campani G. (2016), Antropologia di genere, Torino: Rosenberg\&Sellier.

Campani G. (compiladora) (2010). Genere e Globalizzazione, Pisa: ETS.

Capecchi S. (2006), Identità di genere e media, Roma: Carocci.

Carabí, Á., Segarra, M. (Eds). (2000). Nuevas masculinidades. Barcelona: Icaria.

García, A., Freire, M. (2003). Desarrollo del género en la feminidad y en la masculinidad. Madrid: Narcea.

Heritier, F. (1996). Masculino/ Femenino. El pensamiento de la diferencia. Barcelona: Ariel.

Lomas, C. (2004). Los chicos también lloran: identidades masculinas, igualdad entre los sexos y coeducación. Barcelona: Paidós.

Lorente, M. (2009). Los nuevos hombres nuevos. Los miedos de siempre en tiempo de igualdad. Barcelona: Destino.

Martin Casares, A. (2006). Antropología de género. Madrid: La cátedra ediciones. 
Monreal, M.C. y Martínez, B. (2010). Esquemas de género y desigualdades sociales. En Amador, L. y Monreal, M.C. (Eds.), Intervención social y género. Madrid: Narcea. pp.73-94

Moya Morales, M. (1993) Categorías de género: consecuencias cognitivas sobre la identidad, Revista de Psicología Social, 8 (2), pp. 171-187.

Moya Morales, M., Páez, D., Glick, P., Fernández Sedano, I., Poeschl, G. (1997) Sexismo, Masculinidad-Feminidad y Factores Culturales. Revista electrónica de Emoción y Motivación, 8 (4), pp. $127-142$.

Palacios, M. (2012). El derecho a la igualdad, Salta (Argentina): EUNSA.

Piccone Stella S., Saraceno C., (compiladoras) (1996), La costruzione sociale del femminile e del maschile, Bologna: Il Mulino.

Robledo, Á., Puyana, Y. (compiladoras). (2000). Ética: masculinidades y feminidades. Bogotá: Universidad Nacional de Colombia. Centro de Estudios Sociales.

Sánchez Palencia, C., Hidalgo, J. (editores) (2001). Masculino plural: construcciones de la masculinidad. Lleida: Universidad de Lleida.

Ulivieri S. (compiladora) (1992) Educazione e ruolo femminile. La condizione delle donne in Italia dal dopoguerra ad oggi, Firenze: La Nuova Italia.

Ulivieri S. (compiladora) (2007) Educazione al femminile. Una storia da scoprire, Milano: Guerini.

Ulivieri S., (compiladora), (2014), Corpi violati. Condizionamenti educativi e violenze di genere. Milano: Franco Angeli.

Valcuende, J., Blanco, J. (Eds.) (2003). Hombres. La construcción cultural de las masculinidades. Madrid: Talasa

Varela, N. (2013) Feminismo para principiantes. Barcelona: B de Bolsillo.

VV.AA., (1988), La masculinidad. La búsqueda de un modelo diferente en el juego de la ambigüedad, El País. Temas de nuestra época, 26; año II, 5 de mayo.

VV.AA., (2011), Non è un paese per donne. Racconti di straordinaria normalità, Milano: Mondadori. 